Cahiers de recherches médiévales

Péché et conscience de soi dans L'Espurgatoire seint Patriz de Marie de France

\title{
Myriam White
}

\section{(2) OpenEdition}

Édition électronique

URL : https://journals.openedition.org/crm/2252

DOI : 10.4000/crm.2252

ISSN : 1955-2424

Éditeur

Honoré Champion

\section{Édition imprimée}

Date de publication : 30 décembre 2005

Pagination : 177-189

ISSN : 1272-9752

\section{Référence électronique}

Myriam White, "Péché et conscience de soi dans L'Espurgatoire seint Patriz de Marie de France »,

Cahiers de recherches médiévales [En ligne], 12 | 2005, mis en ligne le 30 décembre 2008, consulté le 15 décembre 2022. URL : http://journals.openedition.org/crm/2252 ; DOI : https://doi.org/10.4000/crm. 2252

Ce document a été généré automatiquement le 15 décembre 2022.

Tous droits réservés 


\title{
Péché et conscience de soi dans L'Espurgatoire seint Patriz de Marie de France
}

\author{
Myriam White
}

1 On a souvent dit la place que tenaient l'Église et la religion dans la société médiévale et dans l'imaginaire d'hommes pour qui le péché et les tourments de l'enfer étaient des réalités indubitables. La légende du purgatoire de saint Patrick, a d'abord été rédigée en latin par le moine H. de Saltrey, puis abondamment réécrite en vers ou en prose en langue vulgaire à travers l'Europe. Non seulement, elle rappelle l'importance de la faute et de son châtiment dans la représentation du monde du Moyen Âge, mais encore elle utilise le dialogue du religieux et du littéraire, du sacré et du profane, dirait-on volontiers, ou plutôt de deux degrés de perception de la sacralité du monde et de l'homme. En effet, la légende est particulièrement significative car elle participe d'une manière essentielle à la fondation d'un nouveau lieu de l'autre monde chrétien au XII ${ }^{\mathrm{e}}$ siècle : le purgatoire ${ }^{1}$. Dès le texte latin du Tractatus de purgatorio sancti Patricii (entre 1179 et 1185), la majeure partie de la narration est consacrée au voyage d'un chevalier dans l'au-delà : le chevalier Owein, se sentant accablé par le poids de ses péchés et ayant conscience d'avoir gravement offensé Dieu, décide de se soumettre à la très périlleuse pénitence du purgatoire de saint Patrick. Il pénètre donc dans une grotte, qui avait été révélée à saint Patrick en Irlande afin de l'aider à convertir les Irlandais, "pur espoünter les corages/des pecheürs e des nunsages » (Espurgatoire de Marie de France, vv.37-8). Il voyage au purgatoire où il affronte dix terribles tourments. Par la force de sa foi, il parvient à franchir un pont merveilleux, s'élançant au-dessus de l'entrée de l'enfer, jusqu'à l'huis du paradis terrestre qu'il visite, guidé par de bienheureux archevêques qui le conduisent jusqu'à la porte resplendissante du paradis céleste. L'histoire du chevalier s'appuie très nettement sur un imaginaire chrétien et nourri des interrogations religieuses contemporaines. Il n'en demeure pas moins que nombre des réécritures - dont la moins méconnue est L'Espurgatoire seint Patriz de Marie de France ${ }^{2}$, qui servira de point de référence ici -, font plus ou moins radicalement glisser le voyage d'Owein vers une aventure chevaleresque telle que celles 
que l'on raconte dans les romans de chevalerie. Pourtant, quel que soit le statut revendiqué par le texte, une question demeure cruciale: celle du péché et de la culpabilité du voyageur.

\section{La punition de certains péchés}

2 L'une des différences entre la légende du purgatoire de saint Patrick et certains romans allégoriques ou autres descriptions de l'enfer, voire du purgatoire comme chez Dante, est l'indétermination quant à la nature des péchés qui sont punis. C'est seulement dans la réécriture en langue anglaise du manuscrit Ashmol $43^{3}$, surnommé Owayn Miles 1, en ce point infidèle à sa source, que les tourments sont les sanctions de péchés précis. On trouve par exemple l'orgueil, la lubricité, la gloutonnerie, la convoitise ou la paresse. En français, il faut attendre la réécriture indirecte et tardive de la légende dans Guérin Mesquin ${ }^{4}$ pour que le héros visite le purgatoire de saint Patrick et que chaque lieu qu'il traverse corresponde à la purgation d'un péché particulier. Pourtant, dès le texte latin, le déroulement de certains tourments semblent faire allusion à des péchés précis. On peut deviner une référence à la gourmandise ${ }^{5}$ dans plusieurs tourments. Lors du deuxième, les suppliciés "pur l'anguisse de lur dolur,/mangerent la terre a tristur " (L'Espurgatoire de Marie de France, vv. 949-50). Le fait de se nourrir ou d'être nourri de choses immondes est un topos quand on évoque la punition de la gourmandise ${ }^{6}$. De plus, lors du cinquième tourment, on utilise le vocabulaire de la cuisine pour évoquer les tourments :

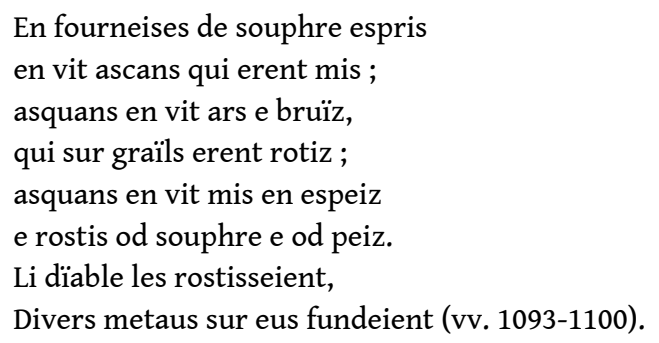

3 D'autres tourments évoquent la luxure ou l'impudicité ${ }^{7}$, notamment par la nudité de tous les suppliciés (v. 946...) On pense également à la maison des bains du septième tourment, qui n'est pas sans évoquer les lieux de prostitution ${ }^{8}$ du Moyen Âge. On trouve également, au cinquième tourment, des suppliciés suspendus à des crochets par différentes parties du corps :

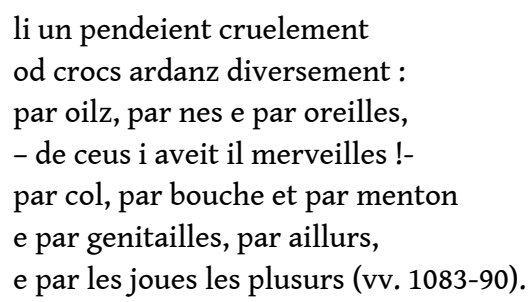

4 On peut imaginer un lien entre la partie du corps par laquelle le supplicié est suspendu et le péché qu'il a commis: la luxure serait alors punie d'une suspension par les "genitailles », par exemple. Il est également loisible d'imaginer dans cette torture la punition d'autres péchés, en lien avec les différentes parties du corps énumérées. Une dislocation du corps s'opère, notamment des traits du visage, ce qui produit une forme de déshumanisation des malheureux. De plus, le fait de suspendre quelqu'un par le cou peut souligner qu'on le dépossède de sa volonté propre: il est condamné à courber 
l'échine. Cela peut être lu comme une condamnation de l'orgueil' . La dilution des traits du visage peut stigmatiser toutes les tricheries avec l'expression du faciès: le mensonge, la coquetterie...

Le fonctionnement du tourment peut suggérer une proportion entre le péché et le châtiment. Ainsi, au septième tourment, on précise que les suppliciés sont plongés dans les bains de métal en fusion plus ou moins profondément :

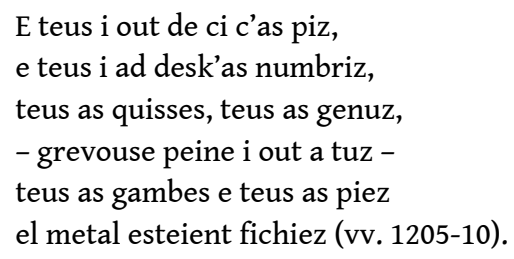

6 C'est encore le cas dans l'image de la roue du sixième tourment qui «tant alout isnelement » (v. 1156). Cette roue plonge plus ou moins les suppliciés dans les entrailles de la terre ou au contraire les élèvent dans un vaste brasier. Elle semble châtier les hommes de leur orgueil en soulignant la vanité de toutes choses, comme la roue de la Fortune, en les invitant à davantage d'humilité. Il est aussi possible de voir une allusion aux richesses de ce monde dans les nombreuses références aux métaux en fusion qui baignent et ébouillantent les malheureux. De surcroît, cette utilisation du métal chauffé se superpose à la référence très péjorative au forgeron qui martèle le corps des hommes : «li autre dïable teneient/maces de fer, sis debatoient» (vv.1101-2). Ainsi, bien que les tourments purgent du péché, sans qu'on précise duquel, certaines peines peuvent être interprétées en elles-mêmes.

7 Par ailleurs, d'autres passages de la légende sont très nettement explicites et condamnent fortement des péchés précis. On trouve notamment la question de l'avarice à chaque fois que la légende mentionne la nécessité de faire l'aumône aux pauvres, afin d'alléger le fardeau de ceux qui sont au purgatoire :

Ceus qui par lius sunt en torment

erent delivres veirement

par messes e par oreisons

e par almones e par dons

qu'om done a povre gent pur eus (vv. 1441-5)

8 Il est question de la faute que constitue le manque de générosité dans l'un des récits secondaires ${ }^{10}$ proposé par le texte latin pour appuyer la signification du voyage d'Owein en montrant les méfaits des démons et la porosité de notre monde à l'autre : deux clercs demandent l'aumône à un paysan qui la leur refuse par cupidité ${ }^{11}$. Les récits secondaires condamnent aussi explicitement le désir charnel coupable. L'évêque Florencien rapporte les mésaventures d'un ermite qui était tenté par les démons qui

$$
\begin{aligned}
& \text { en semblance de femmes nues } \\
& \text { se mustre(re)nt, ke la sunt venues } \\
& \text { pur lui deceivre e engigner } \\
& \text { e feire sun porpos lesser (vv. 2111-4) }{ }^{12}
\end{aligned}
$$

9 Dans le troisième récit d'un ermite, rapporté par un chapelain, les démons ont fait adopter une petite fille à un prêtre. Quand elle sera adolescente, le malheureux sera tourmenté par le désir ${ }^{13}$. La légende a recours aux figures féminines avant tout pour incarner la tentation et la chute de l'homme vertueux. 
d'autres : homicidium dampnabile esse peccatum ${ }^{14}$ (p. 96 $)^{15}$. Or, c'est le péché qui est associé à la figure du protagoniste chevalier, homme d'armes, de combat et de batailles, point sur lequel insistent toutes les réécritures : «Cil qui devant fud bien armez/d(e)'armes de fer e aturnez » (vv. 651-2). Même si on ne le précise pas dans toutes les réécritures, le protagoniste est suspecté d'homicide par sa condition même. Or un récit préliminaire à l'histoire du voyage du chevalier Owein, destiné à présenter la difficulté du ministère de saint Patrick en Irlande énonce la gravité de l'homicide. En effet, un vieil Irlandais désireux de prendre la communion se confesse préalablement à saint Patrick :

Tute dist sa cunfessïun, n'i parlad rien d(e)'occisïun ; n'ert pas pechié, ço lui ert vis, si il aveit homë occis.

Seinz Patriz lui ad mult enquis Se il en aveit nul occis.

Il respundi : « Cink en ai morz, Quel ke ço est ou dreiz ou torz, E mult navrez, mes ne sai mie S'il turnereient puis a vie. Ne quidai pas, bien le sachez, Ke ço fust dampnables pechez.» Li seinz Deu lui mustra e dist Ke ç(o)'ert encontre Jhesu Crist

$E$ ke mult en aveit perdu Sun Creatur e offendu (vv. 235-50)

L'importance de ce passage est rendue sensible par l'emploi du discours direct qui met en valeur les propos du vieil Irlandais. Sa naïveté renforce la gravité de son péché par contraste. La première homélie de la version longue du Tractatus évoque la gravité intrinsèque de tout péché : quod ergo peccatum audet quis dicere parvum? Deum enim exhonorare, quis sane mentis dicturus est parvum $?^{16}$ À plus forte raison, l'homicide est criminel. De manière générale, les péchés explicitement exposés dans la légende renvoient l'image d'un monde plutôt masculin, d'une société à la fois chevaleresque et chrétienne qui se lit également dans une littérature du Graal. On pense à Perceval oublieux de Dieu, rappelé à l'ordre par l'ermite, à Lancelot indigne de sa quête en raison de son amour adultère pour Guenièvre, alors que seul le pur Galaad pourra accéder au Graal. La vision du monde qu'exprime la légende peut ainsi, au moins partiellement, expliquer la tendance des réécritures à tirer cette légende vers la littérature chevaleresque, plus ou autant romanesque que pieuse.

\section{Pénitence et conscience de soi}

Cependant, même si l'on peut deviner la stigmatisation plus ou moins appuyée de certains péchés précis dans la légende de saint Patrick, il demeure que ce n'est visiblement pas l'objectif premier de celle-ci. Il semble en effet qu'elle cherche avant tout à démontrer que la souffrance est nécessaire à la purgation de la faute :

Li grant mesfait de ses pechiez,

dunt ses cors ert pleins e chargez

ne reduta mie a suffrir

peine e turment pur Deu pleisir (vv. 647-50) 
Le chevalier Owein réclame pour sa propre pénitence la plus grande épreuve :

Trop ai forfait a mun Seignur

e offendu mun Creatur.

Pur ç(o)'eslirai, par Deu licence,

La plus grïeve penitence :

A l'Espurgatoire en irrai

Seint Patriz e la enterai,

Ke jo seie de mes pechiez

E delivres e espurgiez (vv. 533-40)

C'est l'homme dans sa totalité, à la fois le corps et l'âme qui doivent souffrir pour permettre la purification: la légende se détache des récits de vision car le chevalier Owein a voyagé dans l'autre monde dans son corps et en vie. Le lien qui existe entre le corps et l'âme est d'ailleurs particulièrement patent dans le déroulement du troisième tourment. Les suppliciés sont livrés à des créatures maléfiques comme des serpents, des dragons ou des crapauds gigantesques qui s'emploient à leur arracher le cœur :

od lur langues qui sunt fuïnes,

percent lur cors e lur peitrines ;

of l'agüesce traient fors,

ço lui ert vis, les quers des cors.

Crapouz i vit, merveilles granz,

Ço lui ert vis, trestuz ardanz.

Sur les piz des asquanz seient,

Od lur becs qu(e)'horribles aveient,

A grant force erent ententis

De traire les quers des chaitis (vv. 1003-12)

Ce n'est pas par hasard que ces créatures s'attaquent au cœur des malheureux : siège des sentiments et notamment de l'amour, il permet les liens entre les hommes et surtout entre l'homme et Dieu. Toute communion avec Dieu devient impossible aux pécheurs, privés de cœur ${ }^{17}$.

Mais dans le mouvement général des réécritures vers la littérature romanesque, la souffrance devient esthétique. Dans le texte latin, déjà, une délectation dans l'exposé de la souffrance est sensible. Les châtiments s'appuient sur des topoï mais se caractérisent par leur compilation, leur accumulation et leur combinaison. On exhibe une souffrance qui ne semble jamais aller jusqu'à satiété: ce sont les clous innombrables qui recouvrent entièrement le corps (quatrième tourment), les suppliciés qui s'élèvent comme autant d'étincelles dans un grand feu démoniaque (neuvième tourment)... On semble se délecter d'une souffrance à la fois itérative et variée. Ce plaisir paradoxal, pris à la narration ou à la lecture de la souffrance, s'appuie sur la croyance en sa fonction rédemptrice, dans une idéologie chrétienne doloriste, et sur le fonctionnement de la littérature comme catharsis. La lecture se substitue alors aux souffrances réelles. Toutefois, un tel fonctionnement, aussi banal qu'il paraisse, modifie en fait radicalement le statut de la légende. Alors qu'elle se présente originellement comme un objet de croyance, invitant le lecteur à une pénitence et à un éventuel voyage au purgatoire de saint Patrick, elle devient par la suite un objet littéraire, dans lequel le plaisir du texte s'écarte de la vie réelle. Le lien entre le texte et la vie se fissure mais cela permet l'émancipation de ce que l'on nommera la littérature. On peut lire au lieu d'aller au purgatoire, alors que l'une des caractéristiques premières de la légende est opposée. Il s'agissait précisément d'assigner une entrée terrestre à l'autre monde, afin qu'il prenne place dans le réel. Au fil des réécritures, l'autre monde se cantonne au 
texte. Mais peut-on pour autant parler d'un repli en littérature? N'est-ce pas plutôt une toute autre ouverture? N'est-ce pas la garantie du déploiement d'un au-delà plus vaste et plus riche encore que celui que le monde géographique peut offrir?

Or c'est par son altérité que le purgatoire de saint Patrick peut répondre à certaines des attentes du public médiéval et susciter l'engouement dont témoignent les abondantes réécritures. Le purgatoire vient souvent satisfaire un besoin de justice que notre monde ne sait contenter. Le purgatoire de saint Patrick présente la particularité de constituer une interface entre ce monde et l'autre et par là, entre l'humain et le divin. Or une telle communication entre l'ici-bas et l'au-delà semble, dans une certaine mesure, rechercher dans l'au-delà les compensations ou les corrections des imperfections de l'ici-bas. Et, si l'on parle du purgatoire et du paradis terrestre, l'au-delà permet de parfaire la justice humaine, qui peut être partiale, erronée ou fautive, grâce à une justice divine, nécessairement idéale. Déjà, saint Patrick avait établi ce que Marie de France nomme la «lei Deu», au vers 196 de L'Espurgatoire. On pouvait donc juger du juste et de l'injuste, du droit et du tort, en fonction de critères divins. H. Bloch ${ }^{18}$ montre que la légende du purgatoire de saint Patrick permettait de moraliser le lien social en en éliminant la violence. Selon lui, elle tente de transformer une société de vendetta (la faide), en se référant à des critères de responsabilité, plus individuels que collectifs. En effet, on note des efforts de lutte contre le meurtre, dans un but de pacification, depuis Guillaume le Conquérant, notamment dans le mouvement de paix normand et irlandais. Le purgatoire a un aspect égalitaire puisque l'on y trouve des personnes de tous âges, des deux sexes et de diverses conditions, comme l'affirme le Tractatus ${ }^{19}$. Dans ce sens, la conception d'un autre monde où l'on purge ses péchés et où tous sont punis proportionnellement à leurs fautes ${ }^{20}$ ne peut que servir une meilleure paix sociale : justice sera rendue un jour à tous et pour tous ! Chacun recevra son salaire!

tels a(s) greignurs, tels a(s) menors,

solum les ovres des plusors.

Cil qui plus peccherent el munt

Greignurs tormenz iluec avrunt (vv. 1732-6)

18 Tous reçoivent « solum ço k'[ils] unt ovré » (v. 59), « seluns iço ke fait [unt] » (v. 1726). Par là, il semble exister des rapports entre justice terrestre et justice divine: l'utilisation du feu au purgatoire ne parait pas sans analogie avec l'ordalie. J. Le Goff remarque que « le feu du Purgatoire a été à la fois un châtiment, une purification et une ordalie, ce qui est conforme au caractère ambivalent du feu indo-européen $» \cdot{ }^{21}$ De plus, «dans les Apocalypses et les visions médiévales le voyage dans l'au-delà doit le plus souvent s'achever avant l'aube, avant le premier chant du coq. Ce sera le cas dans le Purgatoire de saint Patrick où cette exigence de temps fait partie du système de l'ordalie. $»^{22}$ Celui qui entre dans la fosse doit y rester " un jur e une nuit entiere ", comme le rappelle le vers 317 , c'est-à-dire le temps d'un cycle, l'espace d'une nouvelle naissance du jour. L'homme purifié peut à son tour renaitre par analogie du microcosme avec le macrocosme. Mais le feu du purgatoire n'est pas ordinaire car il ne comporte pas l'idée de lumière. M.-M. Davy explique que «le feu brillant évoque la puissance vivifiante de Dieu. Le feu noir s'oppose à l'autre en ce qu'il est obscur et ne brille pas. Il se tient sous le pouvoir du premier et désigne le feu du jugement et de la géhenne (judicalis et gehennalis ignis ${ }^{23}$ ) qui consume le mal. Honorius Augustodunensis, dans l'Elucidarium ${ }^{24}$ parle de ce feu matériel qui, tout en étant privé de lumière, l'emporte autant sur le feu matériel que le feu réel sur l'image peinte du feu. Déjà saint Grégoire, dans ses Moralia(23), avait parlé du feu de l'enfer qui n'éclaire pas ${ }^{25}$. Le feu 
du purgatoire est, par conséquent, un feu de jugement dont la nature paradoxale est conforme à son appartenance à un autre monde ténébreux. Le châtiment des fautes n'est plus seulement le salaire du péché mais un moyen de justice au niveau politicosocial.

Toutefois, l'homme ne peut en aucun cas comprendre tout à fait la justice divine, comme le rappelle le passage consacré à la référence à saint Grégoire et saint Augustin : certum est alios aliis plus minusve pro meritis cruciari; que quidem ab hominibus non possunt diffiniri, quia ab eis minime possunt sciri ${ }^{26}$ (p. 86). Il semble invraisemblable qu'un pécheur puisse se laver de ses fautes en ne faisant qu'un bref voyage de vingt-quatre heures au purgatoire, même si, ce faisant, il franchit les frontières de la vie et de la mort. En effet, les malheureux qu'il y rencontre y restent pendant un temps proportionnel à la quantité et à la gravité de leurs péchés :

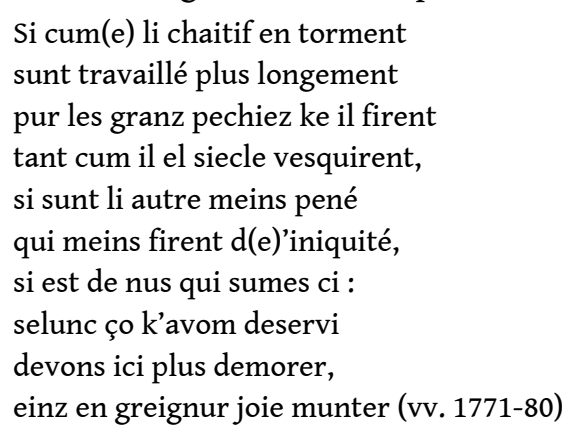

20 Cette prise en compte des péchés de chacun dans la purification indique que la problématique de la faute s'appuie sur une conscience aiguë de la culpabilité de chacun et de la responsabilité individuelle et différenciée. Une conception renforcée de la prière et de la confession comme des moyens d'expression plus individualisés entre l'Eglise, voire Dieu, et le croyant témoigne de cette même évolution.

21 C'est le sens profond de la culpabilité du chevalier qui détermine son voyage dans l'audelà. Il semble que ce soit dans sa confrontation au danger et à un monde d'une altérité radicale que le personnage prenne conscience de lui-même et de sa mesure. Le voyage dans l'autre monde peut être pensé comme un moyen de se découvrir soi-même. Percevoir l'unité du monde, y compris celle de notre monde et de l'autre, par cette entrée terrestre, permettrait également de comprendre l'unité de l'être qui se met en accord avec sa conscience par la pénitence mais aussi l'unité du réel et de l'imaginaire dans la psyché humaine. Le voyage dans l'au-delà serait alors une forme de célébration des retrouvailles de soi avec soi, d'harmonie de soi, impossibles dans notre monde. C'était également dans le sentiment de sa culpabilité que le vieil Irlandais venu se confesser à saint Patrick accédait véritablement à sa propre humanité et sortait de son état de sauvage. Une hiérarchisation morale paraît exister entre les hommes, dans une conception chrétienne de l'existence : plus le sentiment de culpabilité est grand, plus la valeur morale est certaine. L'autre monde guette les faillites du nôtre, c'est pourquoi on invite le lecteur à garder en mémoire les images qui ont été évoquées et dans ce sens, les souvenirs de lecture ont un fonctionnement analogue à celui de certaines visions, si l'on en croit A. J. Gurevich, selon qui «la vision se présentait comme une sorte de tentative pour analyser la personnalité; l'Enfer et le Paradis sont aux prises à l'intérieur de l'âme humaine ». Paradis et enfer sont aux bornes de la conscience et "l'étendue de la vision, c'est avant tout l'extériorisation de l'étendue de l'âme de l'homme au Moyen Âge $\aleph^{27}$. De fait, il s'agit bien d'une responsabilité personnelle de 
chacun dans la légende du purgatoire de saint Patrick. Chacun répond de ses actes. « $\mathrm{Ce}$ ne sont déjà plus les démons accusateurs ni les anges protecteurs qui jouent le rôle principal dans ce jugement, mais la conscience de l'inculpé lui-même $»^{28}$. La conscience de soi passe donc par une profonde culpabilité.

Toutefois, la plénitude de la découverte de soi est liée de façon plus positive avec la nécessité de la foi. En effet, le chevalier doit non seulement profondément ressentir la gravité de sa faute afin de trouver en lui-même les ressources pour devenir meilleur mais il doit aussi savoir s'en remettre à Dieu. Ainsi, pendant le voyage au purgatoire, l'issue positive de chaque tourment pour le chevalier dépend de l'invocation du nom de Dieu : à partir du moment où l'homme confie son sort à son Seigneur, il est vainqueur. S'il oublie sa dépendance par rapport à Dieu, il risque de perdre son être, comme au neuvième tourment quand le chevalier pense in extremis à l'invocation salvatrice en raison de la douleur qu'il endure :

Tant fud de cel torment hastez,

pur poi k'il ne s'ert obliez

de nomer le non sun Seignur ;

puis le noma par grant dulçur (vv. 1297-1300)

D'ailleurs beaucoup d'autres hommes qui ont tenté le voyage ne sont jamais revenus, comme le rappelle la légende :

Mult i sunt entreé e perdu,

ne sout hom k'il sunt devenu.

Kar n'orent pas ferme creance,

Bone fei ne dreite esperance,

Ne porent suffrir les turmenz,

Pur ço remistrent il dedenz.

Par les granz turmenz ke il virent

Deu oblierent e perdirent (vv. 599-606)

Les malheureux qui se sont perdus au purgatoire de saint Patrick ont manqué de foi ou se sont méconnus. Ils n'ont pas su reconnaître le grand besoin qu'ils avaient de Dieu et la nécessité de la foi pour traverser l'au-delà. Ne pas rechercher assez vivement le soutien divin est la manifestation de la faute d'orgueil d'un homme qui présume de ses forces et se fait piéger par ses propres faiblesses qu'il méconnaît.

De plus, une telle ignorance de soi-même est une forme de péché. Cependant, le choix d'un héros chevalier, confronté à ses très lourds péchés ne doit pas faire oublier qu'il n'est qu'un laïc bien imparfait, ce qui fait d'ailleurs décider à certains auteurs de réécritures de le faire changer d'état et devenir religieux à la sortie de la fosse ${ }^{29}$, ce qui n'est absolument pas le choix de la source ou de Marie de France qui insistent sur la possibilité de faire son salut en tant que laïc : « e li reis lui ad respondu/chevaliers seit, si cum il fu " (vv. 1927-8). Néanmoins, le protagoniste est une figure de pécheur repenti, tout comme le vieil Irlandais homicide. A contrario, on trouve plusieurs figures de religieux pécheurs dans la légende et elles renforcent la culpabilité qui pèse sur les hommes car même ceux qui devraient se garder du mal succombent aux tentations. On pense par exemple aux personnages des récits courts qui suivent le voyage du chevalier Owein et appuient son propos : le faux ermite ou le prêtre tenté par une orpheline. Ces figures viennent en contrepoint des figures grandies du prieur qui n'a plus qu'une dent $^{30}$ et qui attend la mort avec sagesse comme une promesse, de saint Patrick patient face aux pécheurs Irlandais ou de Gilbert ${ }^{31}$, compagnon fidèle du chevalier Owein... Les religieux, personnages valorisés, sont la preuve de l'omniprésence du mal et de sa 
puissance, comme de la grâce qu'offre la foi en Dieu. La faute et le péché ne sont pas les mêmes suivant le degré de conscience qu'on en a, les religieux qui devraient aspirer au Bien sont plus condamnables encore que les laïcs insensés: cette idée est particulièrement efficace pour la constitution d'une conscience individuelle et d'un sujet, ce qui permet de prendre en compte l'intention ${ }^{32}$ qui préside à une action, non plus l'acte seul.

De fait, la légende du purgatoire de saint Patrick paraît se situer à un moment privilégié dans la conquête d'une identité individuelle dans un Moyen Âge qu'on dit si souvent collectif. En effet, le péché tel qu'il se dessine dans les récits consacrés au voyage du chevalier Owein semble consister en un déséquilibre entre le désir et la crainte au sein de la conscience du sujet. Le dernier tourment que rencontre le chevalier Owein au purgatoire le symbolise particulièrement bien. Les démons poussent le chevalier à s'engager sur un pont qui s'élance au-dessus d'un fleuve effrayant qui n'est autre que l'entrée de l'enfer, mais qui peut également le conduire au paradis terrestre. Le pont lui-même est triplement périlleux: il est glissant, "escolurgables" (v.1353), passablement étroit et extrêmement élevé au-dessus du fleuve. La peur fait redouter de s'y engager. Mais lorsque le chevalier trouve le courage d'y avancer, le pont magique se transforme, "tant cum [le chevalier] plus alad avant/e plus s'alad asseürant» (vv. 1377-8), si bien qu'il parvient à atteindre la rive paradisiaque du fleuve sans plus d'encombres. L'absence de peur de certains hommes qui souhaitent pénétrer dans la fosse sans l'autorisation de la communauté religieuse qui en a la garde est un péché. Mais une peur plus puissante que le désir en est également un. Il s'agit donc pour le croyant de rechercher une juste crainte et une puissante confiance en Dieu. La portée positive de la légende réside d'ailleurs dans l'importance qu'elle accorde non seulement à la recherche et la conquête du salut mais à la revendication d'une forme de quête du bonheur, de la félicité. Ne pas rechercher le salut et la joie en Dieu serait le péché le plus grave: il ne s'agit pas d'accabler l'homme sous le poids de sa faute mais de lui offrir une alternative radicale et profonde au péché dans la jubilation de la certitude du salut. La légende du purgatoire de saint Patrick n'invite pas à un repli total du désir mais au contraire conduit à son épuration. C'est l'un des messages du christianisme : il faut rejeter les mauvais désirs, ceux des choses humaines, mais rechercher avec ardeur les biens spirituels. Ainsi, la légende se livre à une exaltation du désir du voyage, de la purification et de Dieu. Le chevalier affirme très fortement purgatorium sancti Patricii subire volo ${ }^{33}(\mathrm{p} .124)$. Le texte latin fait retentir la volonté impérieuse par l'emploi du verbe volo. Owein élit sa pénitence :

Li chevaliers pur ses pechiez

fud mult tristes e esmaiez ;

pense ke digne penitence

fera solum la Deu consense (vv. 521-4)

Il refuse de s'en laisse détourner alors que tous tentent de le dissuader: «tant ert fervenz en sun desir/ne l'en puet li priurs partir » (vv. 573-4),

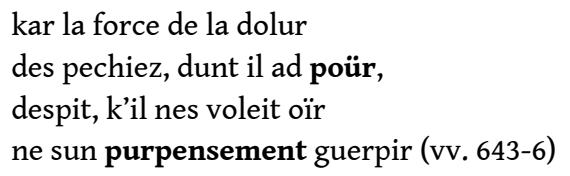

Quand il parvient au paradis terrestre, on confirme que «bien ad empli sun/qui en tel liu lui fist venir » (vv. 1529-30). La peur et le désir s'équilibrent dans la juste conscience de soi. Le Moyen Âge enseigne ici une grande hauteur de vue, qui invite à une 
redécouverte de l'ardeur de certains désirs fondamentaux. La seconde homélie du texte latin du manuscrit de la British Library montre combien il faut désirer le Bien suprême: Denique si multe et magne delectationes sunt in rebus delectabilibus, qualis et quanta est delectatio in illo qui fecit illa delectabilia ! 0 qui hoc bono fruetur, quid illi erit, et quid illi non erit? Certe quicquid volet erit, et quod nolet non erit. Ibi quippe erunt bona corporis et anime, qualia nec oculus vidit, nec auris audivit, nec cor hominis cogitavit. Cur ergo par multa vagamur querendo bona anime nostre et corporis nostris? Amemus unum bonum in quo sunt omnia bona, et sufficit. Desideremus simplex bonum, quod est omne bonum, et satis est. Quid enim amas caro, quid desideras anima? Ibi est, ibi est quicquid amatis, quicquid desideratis! ${ }^{34} \mathrm{Ce}$ Moyen Âge rappelle que trouver les ressources pour devenir meilleur est un but délectable.

Chacun est face à son péché mais surtout face à lui-même dans la quête de la félicité. Néanmoins, la légende n'abandonne pas si simplement et strictement chacun à sa peine. Il est des adoucissements possibles dans la foi, donc par Dieu, comme le prouve l'utilisation de l'invocation salvatrice au cours du voyage du chevalier Owein. Mais encore, la légende du purgatoire de saint Patrick fait référence aux croyances dans la prière pour les morts ${ }^{35}$, qui sous un certain angle, est une manifestation de la cohésion de la société et de la compassion entre les hommes. Le chevalier Owein lui-même demande que l'on prie pour lui, selon le vers 661 de L'Espurgatoire de Marie de France: « il dist a tuz: "Preez pur mei" ». Les vivants peuvent et doivent prier pour les morts. Cette croyance en l'intercession des vivants pour les morts sous-entend une action des suppliques humaines sur la justice divine. Elle renforce les liens entre les hommes à l'image de celui qui unit les hommes et Dieu, comme le rappelle la seconde homélie du Tractatus du manuscrit de la British Library: Et utique quoniam quantum quisque diliget aliquem, tantum de bono ejus gaudet, sicut in illa perfecta felicitate unusquisque plus amabit sine comparatione Deum plus quam se et omnes alios secum, ita plus gaudebit absque estimatione de felicitate Dei, quam de sua et omnium aliorum secum. Sed si Deum sic diligent toto corde, (tota mente), tota anima, ut tamen totum cor, tota mens, tota anima non sufficiat dignitati dilectionis, profecto sic gaudebunt, toto corde, tota mente, tota anima, ut totum cor, tota mens, tota anima non sufficiat plenitudini gaudii ${ }^{36}$. Elle est vecteur d'un grand espoir dans la solidarité humaine et dans l'efficacité de la charité, de la compassion ou du pardon, formes variables de l'amour, qui transcenderaient la frontière de la vie et de la mort. Or cet amour permet de dépasser la culpabilité, le péché et la souffrance, pour s'élever vers la joie parfaite.

\section{NOTES}

1. Jacques Le Goff, La Naissance du purgatoire, Paris, 1981, rééd. Gallimard, coll. Folio/Histoire, 1991.

2. Ed. Y. De Pontfarcy, Peeters, Paris-Louvain, 1995.

3. C. Horstmann, Altenglishe Legenden, Paderborn, 1875.

4. Guerin Mesquin, trad. de l'italien par Jean Decuchernmoys, 1530. 
5. Il est question de ce péché dans la première homélie du texte latin contenu dans le manuscrit British Library, Royal 13 B viii (R) : nos obedientes gule diversa fercula querimus, et ipse inedia pro nobis afficitur (G. Lachin, Il Purgatorio di San Patrizio, Maria di Francia, Roma, Carocci, Biblioteca medievale, 88, 2003, p. 316, 176), « Nous, obéissants à la gloutonnerie, nous recherchons des mets divers, et lui est privé de nourriture pour nous » (ma trad.).

6. Voir à ce sujet, dans la postérité de la légende, le voyage de Guérin Mesquin au purgatoire de saint Patrick, et particulièrement les gravures du livre VI, de Guérin Mesquin, trad. de l'italien par Jehan Decuchermoys, 1530.

7. Cette faute est mentionnée dans la première homélie du texte latin contenu dans le manuscrit British Library, Royal 13 B viii (R): Nos ad illicitam arborem rapit concupiscentia, ipsum perfecta caritas pro nobis ducit ad crucis supplicia. Nos presumimus vetitum, et ipse subit eculeum. Nos jocando delectamur cibo, et ipse condolendo nobis laborat in patibulo. Nobis nam lasciviens conridet Eva, ipsi vero plorans compatitur Maria (G. Lachin, Ibidem, p. 316, 177-180), «Nous, la concupiscence nous entraîne vers l'arbre défendu, Lui, sa charité parfaite l'a conduit pour nous au supplice de la croix. Nous avons l'audace de transgresser l'interdit, et c'est lui qui subit le tourment. Nous avons le plaisir de manger en plaisantant, et lui souffre sur le bois en compatissant pour nous. Car, Eve, folâtre, rit avec nous, mais Marie en pleurs souffre avec lui » ( ma trad.).

8. Cf. J. Rossiaud, La prostitution médiévale, Champs Flammarion, 1988.

9. Ce péché est clairement évoqué dans la première homélie du texte latin contenu dans le manuscrit British Library, Royal 13 B viii (R) : Nos superbimus, ipse humiliatur! Nos tumemus, ipse attenuatur! Nos prelatis nostris inobedientes sumus, ipse patri suo obediens, scelus luit inobedientie nostre (G. Lachi, op.cit., p. 316, 173-175), " Nous sommes fiers, Lui est humilié! Nous nous enflons d'orgueil, Lui est rabaissé ! Nous désobéissons à nos supérieurs, Lui, obéissant à Son Père, expie le forfait de notre désobéissance » (ma trad.).

10. On trouve deux versions du texte latin: une version courte, celle du manuscrit British Library Harley 3846, et une version longue, celle du manuscrit British Library Royal 13 B viii. La première paraît antérieure à la seconde. La version longue se caractérise notamment par l'addition de récits en appendice, rapportés par l'évêque Florencien et son chapelain. Marie de France réécrit vraisemblablement un Tractatus composite : Elle parait traduire une version courte mais rapporter la plupart des additions de la version longue. Ces homélies sont assez proches de celles du texte latin court.

11. Ce récit n'est repris que par deux réécritures en vers : celle de Marie de France (vv. 2147-80) et la réécriture anonyme du manuscrit Cotton Domitianus A iv (P. De Wilde, Le Purgatoire de saint Patrice : édition de la version en vers anglo-normande du manuscrit British Library Cotton Domitianus IV, Thèse, Univ. d'Anvers, 1990-91, vv. 1517-50).

12. Ce récit n'est repris que par les mêmes versions que précédemment (respectivement aux vers 2071 à 2116 et 1445 à 1486).

13. Le récit n'est réécrit que dans les versions de Marie de France (vv. 2181-2296) et du manuscrit Cotton Domitianus A iv (vv. 1551-1688).

14. «L'homicide est un péché qui entraîne la damnation » (ma trad.), Marie de France réécrit « ke ço fust dampnables pechez » (v. 246).

15. Seules les deux versions qui ont repris les récits secondaires ont réécrit la confession du vieil Irlandais (Marie de France, vv. 215-64, et Cotton Domitianus A iv, vv. 57-86).

16. Manuscrit British Library, Royal 13 B viii (R) (G. Lachin, op. cit., p. 312, 147-148) : « quel péché ose-t-on donc appeler petit? En effet, déshonorer Dieu, qui, saint d'esprit, dira que c'est une petite chose?» (ma trad.).

17. On peut également voir une allusion au cœur comme siège de la vie, du souffle vital. Cf. B. Ribémont, «Le cuer del ventre li a trais. Cœur arraché, cœur mangé, cœur envolé : quelques remarques médico-théologiques sur un thème littéraire ", Senefiance, 29, 1992, pp. 347-61.

18. H. Bloch, The Anonymous Marie de France, Chicago, University of Chicago Press, 2003. 
19. Traduit dans L'Espurgatoire, v. 983, 1037, 1199, 1225, 1267...

20. Une forme de calibrage de la peine semble possible, comme au septième tourment où les corps des suppliciés sont partiellement et variablement trempés dans les métaux en fusion. Voir à ce sujet, H. Bloch, op. cit., pp. 277-279.

21. J. Le Goff, op. cit., p. 67.

22. Ibid., p. 41.

23. «Le feu judiciaire et de l'enfer»

24. Y. Lefèvre, L'Elucidarium et les lucidaires. Contribution, par l'histoire d'un texte, à l'histoire des croyances religieuses en France au Moyen Âge, Paris, E. de Boccard/Ecole française d'archéologie d'Athènes, 1954.

25. M. M. Davy, Initiation à la symbolique romane, Paris, 1977, p. 161.

26. «Il est certain que les uns sont tourmentés plus ou moins que les autres en fonction de leurs mérites, qui, certes, ne peuvent être déterminés par les hommes, parce qu'ils peuvent très peu être connus d'eux. "

27. A. J. Gurevich, «Popular and Scholarly Medieval Cultural Traditions : note in the margin of J. Le Goff's book », Journal of Medieval History IX, 1983, pp. 71-90, p. 266.

28. A. J. Gurevich, op. cit., p. 265

29. Voir les réécritures éditées par J. Vising, Le Purgatoire de Saint Parice des Ms. Harléien 273 et fonds fr. 2198, Göteborg, 1916, et C. M. Van der Zanden in Étude sur le Purgatoire de Saint Patrice accompagnée du texte latin d'Utrecht et du texte anglo-normand de Cambridge, Amsterdam, 1927.

30. Il s'agit d'un récit qui précède celui du voyage d'Owein (vv. 383-420).

31. Voir vv. 1913-2056.

32. À ce sujet, voir J.-C. Schmitt, Le Corps, les rites, les rêves, le temps, Paris, Gallimard, 2001, p. 251.

33. "Je veux subir le purgatoire de saint Patrick.»

34. G. Lachin, op. cit., pp. 332-334, 295-303, « si l'on trouve de nombreux et grands plaisirs dans les choses délectables, quelle est la nature et l'intensité du plaisir que l'on trouve dans celui qui a créé ces choses délectables! ô celui qui aura la jouissance de ce Bien, que recevra-t-il et que ne recevra-t-il pas ? Certes, il aura ce qu'il désire et n'aura pas ce qu'il ne veut pas. Certes, là seront les biens du corps et de l'âme, tels que l'œil ne les a pas vus, tels que les oreilles ne les ont pas entendus, tels que le cœur de l'homme ne les a pas imaginés. Pourquoi donc errons nous partout à la recherche de Biens pour notre âme et pour notre corps? Aimons l'unique Bien dans lequel sont tous les Biens, et cela suffit. Désirons le Bien à la nature simple, qui est tout Bien, et c'est assez. En effet, qu'aimes-tu, ma chair, que recherches-tu, mon âme ? Là se trouve, là se trouve tout ce que vous aimez, tout ce que vous désirez !» (ma trad.).

35. À ce sujet, voir Jacqueline Amat, Songes et Visions, L'au-delà dans la littérature latine tardive, Études Augustiniennes, Paris, 1985, p. 407sq. (elle rappelle qu'on allait jusqu'à baptiser les morts). 36. G. Lachin, op. cit., p. 336, 324-325, «Et en tout cas, puisque plus on aimera quelqu'un, plus on se réjouira de son bonheur, de même que dans cette parfaite félicité, chacun aimera plus Dieu que lui-même et que les autres avec lui, et sans commune mesure, de même il se réjouira incomparablement plus de la félicité de Dieu, que de la sienne et de celle de tous les autres avec lui. Mais s'ils aiment Dieu de tout leur cœur, (de tout leur esprit,) de toute leur âme, au point que, cependant, tout leur cœur, tout leur esprit, toute leur âme ne suffise pas à la dignité de leur amour, assurément ils se réjouiront de tout leur cœur, de tout leur esprit, de toute leur âme, au point que tout leur cœur, tout leur esprit, toute leur âme ne suffise pas à la plénitude de leur joie. » (ma trad.). 


\section{AUTEUR}

MYRIAM WHITE

Université d'Artois 